\title{
LARC - Virtual Laboratory for Practice Development in the Discipline of Computer Net- works Integrated with Moodle
}

http://dx.doi.org/10.3991/ijes.v2i1.3671

K. H. A. Ferreira, Universidade do Estado do Rio Grande do Norte - UERN and Universidade Federal Rural do SemiÁrido - UFERSA, Mossoró, Brazil.

R. W. de Lima, Universidade do Estado do Rio Grande do Norte - UERN, Mossoró, Brazil.

J. O. M. Chaves, Universidade do Estado do Rio Grande do Norte - UERN and Universidade Federal Rural do SemiÁrido - UFERSA, Mossoró, Brazil.

M. V. A. de Lima Universidade do Estado do Rio Grande do Norte - UERN and Universidade Federal Rural do SemiÁrido - UFERSA, Mossoró, Brazil.

Abstract - The educational system has moved towards new possibilities for the enrichment of the techniques of teaching and learning, always seeking the good development of the student. Students do not always have a physical space that supports the development of their practices, either by lack of equipment or lack of time in institutions. In this context, this paper presents a Virtual Lab for teaching computer networks integrated with Moodle, allowing the student to use NS2 simulator to validate the scenarios created by the scripting language OTCL.

\section{LARC - Laboratório Virtual para o auxílio de práticas na disciplina de Redes de Computadores integrado ao Moodle}

\author{
K. H. A. Ferreira ${ }^{1,2}$, R. W. de Lima ${ }^{1}$ J. O. M. Chaves ${ }^{1,2}$ e M. V. de A. Lima ${ }^{1,2}$ \\ ${ }^{1}$ Universidade do Estado do Rio Grande do Norte, LORDI, Mossoró - RN, Brasil \\ ${ }^{2}$ Universidade Federal Rural do Semi-Árido, UFERSA, Mossoró - RN, Brasil
}

\begin{abstract}
Resumo-O sistema educacional tem caminhado rumo a novas possibilidades para o enriquecimento das técnicas de ensino e aprendizagem, visando sempre o bom desenvolvimento do aluno. Nem sempre os alunos contam com um espaço físico que dê suporte ao desenvolvimento de suas práticas, seja pela falta de equipamentos ou por falta de horários nas instituições. Neste contexto, este artigo apresenta um Laboratório Virtual para o ensino de redes de computadores integrado ao Moodle, permitindo ao aluno a utilização do simulador NS2 para validação dos cenários criados por meio da geração de scripts na linguagem OTCL.
\end{abstract}

Palavras Chaves-Ambiente Virtual de Aprendizagem, Laboratório Virtual, Moodle, Simulador de Redes.

\section{INTRODUÇÃO}

A crescente demanda dos cursos oferecidos pela modalidade de Ensino a Distância (EaD) juntamente como as novas Tecnologias da Informação e Comunicação (TIC) [1] possibilitam uma nova modalidade de curso a distância através da Internet, denominados de cursos online.

Foi através deste cenário que os Ambientes Virtuais de Aprendizagem (AVA) [2], tomaram forma e foram desenvolvidos para gerir essa nova modalidade de apoio ao desenvolvimento e andamento de cursos.

Em áreas do conhecimento que demandam muitas atividades práticas, como a ciência da computação, engenharia, física e química, o uso de laboratórios é um componente essencial do currículo. A combinação da teoria com as atividades práticas é um elemento de grande importância para que ocorra um aprendizado efetivo [3].

Esses ambientes, repletos de recursos pedagógicos, têm como objetivo auxiliar o professor no processo de criação, acesso, planejamento, controle e desenvolvimento de curso ou disciplina, para ser executado num meio onde a interação professor-aluno possa acontecer à distância, isto é, em ambientes fisicamente distintos [4].

É nesse cenário que surgem os Laboratórios Virtuais, que possibilitam o desenvolvimento de competências técnicas do aluno para melhorar o aprendizado e a fixação do conhecimento teórico adquirido no decorrer do curso. Dessa forma, um Laboratório Virtual promove o acesso a experimentos a partir de um espaço virtual, compensando a falta de interação e a indisponibilidade de horários ou de recursos necessários às experiências práticas [3].

Para o ensino do tema de redes de computadores, é inevitável o uso de experimentações, com vários objetivos imprescindíveis como: localizar o estudante sob a pilha de protocolos; visualizar as características de hosts, enlaces e portas; analisar seu comportamento em diferentes topologias e com cenários distintos.

Devido a isso, este trabalho tem como propósito desenvolver um Laboratório Virtual, LARC, para proporcionar ao aluno de redes de computadores um ambiente atrativo para o desenvolvimento das práticas da disciplina, permitindo ainda, a utilização do laboratório para criação de cenários para o simulador de redes Network Simulator 2 
(NS2) de forma gráfica por meio da geração de scripts na linguagem OTCL.

Para demonstrar melhor este trabalho, este artigo está dividido da seguinte maneira: na Seção II apresentaremos os trabalhos relacionados; a Seção III aborda a estrutura do LARC e a forma de integração com o Moodle; na Seção IV veremos os resultados e discussões; e, por fim, na Seção V serão expostas as considerações finais e trabalhos futuros.

\section{TRABALHOS RELACIONADOS}

Os simuladores possibilitam o desenvolvimento de competências técnicas do aluno para melhorar o aprendizado e fixar o conhecimento teórico adquirido no decorrer do curso. Dessa forma, promovem o acesso a diferentes experimentações compensando a falta de disponibilidade de horários ou de recursos necessários às experiências práticas [5].

Em um contexto aproximado à pesquisa apresentada neste artigo, algumas iniciativas listadas abaixo foram realizadas no sentido de gerar scripts para o NS2 tirando o peso do aluno em dominar as especificações da linguagem de programação OTCL.

O GENESI é um software que tem como proposta central a geração de código fonte para o NS, em OTCL [6]. Possui um gerenciador gráfico para geração dos códigos fontes, com retorno de dados relevantes em forma de gráfico e, consequentemente, a validação de projetos de redes. Seu principal objetivo é auxiliar no processo de simulação [6].

Desenvolvido na linguagem Java e com auxílio da biblioteca Jgraph, o NsGraph foi concebido com o propósito de facilitar a construção e configuração de cenários de simulação de forma automatizada, gerando scripts para o NS2. Porém, o NsGraph não possui visualização gráfica da simulação, tendo que sempre recorrer ao módulo externo de animação Network Animator (NAM) [7].

Outra ferramenta que possui proposta semelhante ao laboratório apresentado com relação à geração de scripts é o TCLGen, um projeto da Universidade de Coimbra, que se dispõe a desenvolver uma interface gráfica em Java para a geração de códigos OTCL [8].

As ferramentas descritas acima são bastante restritas com relação aos cenários de redes que podem ser confeccionados, e não possuem uma interface própria para simulação, tendo sempre que recorrer ao visualizador gráfico NAM.

\section{ESTRUTURA DO LARC E INTEGRAÇÃO COM O MOODLE}

A utilização de Laboratórios Virtuais é essencial para o desenvolvimento das atividades do aluno por meio da junção da teoria com a prática. Este artigo apresenta o LARC que é um Laboratório Virtual para simulação de cenários de redes de computadores que integra um simulador de redes em um AVA, proporcionando ao aluno um ambiente atraente e flexível para criação de cenários simulados.

Utilizando HTML5 e framework jQuery, o laboratório visa atender a um público tanto de ensino presencial quanto aos alunos de cursos à distância. O LARC possibilita ao aluno a criação, graficamente, de cenários de redes e posteriormente executá-los com o NS2. Permitindo assim ao aluno a configuração de um cenário completo utilizando uma interface amigável com o princípio de arrastar e soltar.

Para isso, os cenários gráficos são transformados em scripts OTCL que são repassados ao NS2 por meio de dois agentes reativos [9], como ilustra a Figura 1 . Nesta imagem é possível verificar a integração do LARC com o Moodle e toda a comunicação existente na comunicação com o NS2.

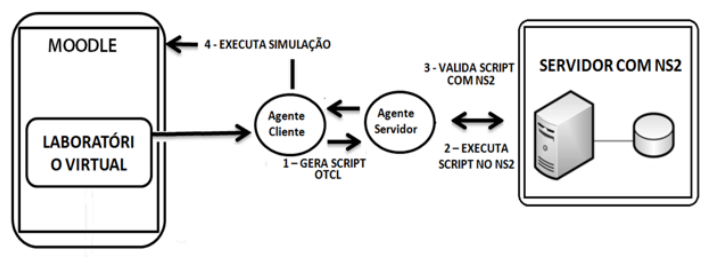

Figura 1. Arquitetura básica do Laboratório Virtual

\section{A. Comunicação com o NS2}

Desenvolvido pela Universidade de Berkeley utilizando as linguagens de programação OTCL e C++, o Network Simulator 2 (NS2) é uma ferramenta dirigida a eventos e trabalha simulando vários tipos de redes TCP/IP. Suporta diversos tipos de tráfegos e pode ser totalmente configurada para se adequar a vários tipos de cenários [10].

Foi inicialmente desenvolvido para ambiente Unix, sendo hoje executado em várias outras plataformas como Linux, SunOS, FreeBSD, Solaris e até mesmo em Windows por meio de emuladores como cygwin [10]. Utiliza duas linguagens de programação para criação dos cenários, a $\mathrm{C}++$ mais robusta para manipulação de bytes, pacotes e para implementar algoritmos que rodem um grande conjunto de dados, e a OTCL para criar o modelo da simulação [10].

A $\mathrm{C}++$, que é uma linguagem compilada e de uso tradicional, mostrou-se mais eficaz na utilização do NS2 [10]. A utilização apenas da linguagem $\mathrm{C}++$ durante o processo de simulação, acarretaria em um desgaste do aluno, pois a cada mudança inserida no cenário desenvolvido, haveria a necessidade de recompilar todo o projeto para testá-lo.

O uso da linguagem OTCL, que é interpretada, evita esse desgaste por parte do usuário, pois há uma simplificação no processo interativo de mudar e reexecutar o modelo [10].

Apesar das características positivas do NS2, o mesmo não possui uma interface amigável ao usuário. Dessa forma, para evitar que o aluno fíque preso à linguagem de especificação utilizada pelo NS2 na criação dos elementos que compõem a rede, o LARC proporciona ao aluno a geração automática de scripts na linguagem OTCL para serem executados com o NS2. Dessa forma, com o LARC, o aluno tem a possibilidade de criar e configurar vários cenários de redes.

Como apresentado anteriormente, a integração do LARC com a ferramenta NS2 é feita com a utilização de dois agentes reativos, um no lado cliente que fica encarregado de ler e repassar os scripts gerados pelo aluno no LARC, e outro agente no servidor, que executa o script no NS2 [9].

Em seguida, de posse dos resultados da simulação, o agente servidor envia um status de "ok" para o agente cliente, que por fim executa a simulação no browser do aluno. 


\section{B. Integração com o Moodle}

O LARC é utilizado como um bloco (block) no Moodle, ou seja, o Moodle possui uma estrutura modular, em que cada bloco tem um conteúdo específico que pode ser editado ou adaptado às necessidades do autor. A Figura 2 ilustra a integração do LARC no Moodle. O professor faz o download do LARC e extrai para o diretório moodle/blocks.

Após a extração do bloco LARC para o diretório blo$c k s$, o professor faz a adição do bloco para o curso de redes, como ilustrado na Figura 3.

\section{Plataforma JADE}

O Java Agent Development framework (JADE) é um ambiente para desenvolvimento de aplicações baseada em agentes conforme as especificações da Foundation for Intelligent Physical Agents (FIPA) para interoperabilidade entre sistemas multiagentes. Foi desenvolvido e suportado pelo CSELT (atual TILAB) da Universidade de Parma na Itália [9].

Segundo [9], o principal objetivo do JADE é simplificar e facilitar o desenvolvimento de sistemas multiagentes garantindo um padrão de interoperabilidade entre esses sistemas por meio de um conjunto de agentes de serviços, os quais tanto facilitam como possibilitam a comunicação entre agentes, de acordo com as especificações da FIPA. $\mathrm{O}$ desenvolvimento de JADE se baseou nos seguintes princípios:

- Interoperabilidade - JADE é aderente ao padrão FIPA, desta forma, agentes em JADE podem operar com quaisquer outros agentes, desde que os mesmos também apresentem conformidade ao mesmo padrão;

- Uniformidade e portabilidade - JADE apresenta um conjunto homogêneo de APIs que são independentes da camada de rede e também do ambiente de execução Java;

- Facilidade de uso - A complexidade do middleware é ocultada através do uso de um conjunto de APIs simples e intuitivo;

Com relação à Filosofia, os programadores não precisam fazer uso obrigatório de todos os recursos providos pelo middleware. O Modelo de Referência FIPA, para Plataforma de Agentes, é composto de:

- Agent - é o agente propriamente dito, cujas tarefas serão definidas de acordo com o objetivo da aplicação. Encontra-se dentro de uma plataforma de agentes (Agent Platform) e realiza toda sua comunicação com os demais agentes através de troca de mensagens, e relacionando-s com aplicações externas (software);

- Agent Management System (AMS) - responsável por gerenciar o ciclo de vida dos agentes na plataforma e prover um serviço de guia de endereços (white pages), cabe a ele manter o diretório de identificadores de agentes (Agent IDs-AID) associando os identificadores lógicos dos agentes à informações de endereçamento e status;

- Directory Facilitator (DF) - que funciona como um serviço de páginas amarelas com capacidades básicas de prover serviços desejados. Uma instância especial deste agente, chamada Default DF, deve estar presente em qualquer plataforma aderente ao padrão FIPA;

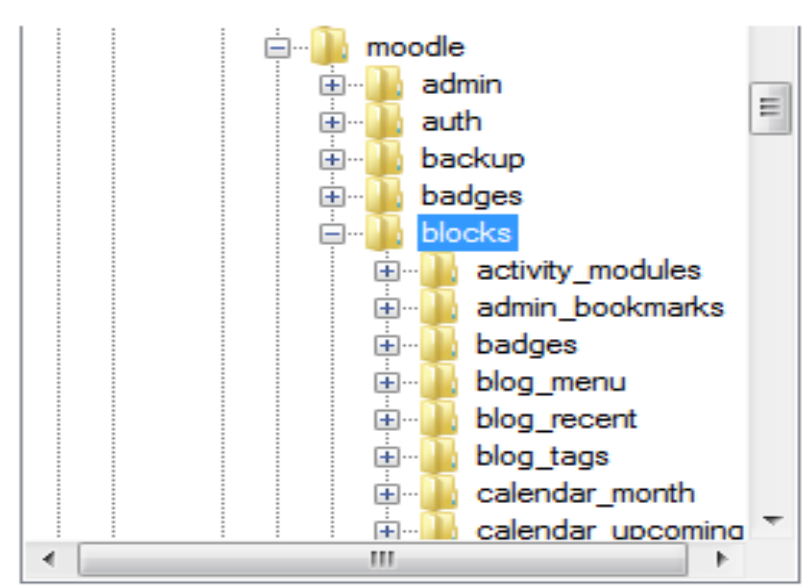

Figura 2. Extraindo o LARC para a pasta blocks

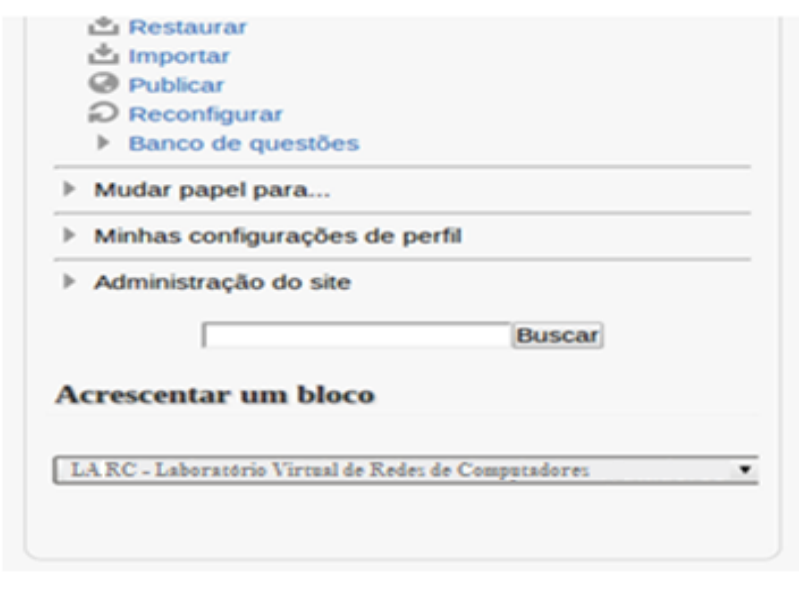

Figura 3. Adicionando o bloco LARC ao Moodle

- Message Transport System (MTS) - também conhecido como canal de comunicação dos agentes (Agent Communication Channel-ACC), é o agente responsável por prover toda a comunicação entre agentes dentro e fora da plataforma.

\section{RESULTADOS E DISCUSSÕES}

O trabalho aqui apresentado visa auxiliar o desenvolvimento das atividades práticas da disciplina de redes de computadores possibilitando um melhor aprendizado do aluno.

A Figura 4 ilustra o LARC integrado ao Moodle. No laboratório o aluno conta com equipamentos como host, $h u b$, switch, roteadores, servidores, entre outros que são comuns nas práticas desenvolvidas em um ambiente real.

Para utilizar o NS2 com o LARC, primeiramente o aluno cria um cenário de redes como ilustrado na Figura 5.

Posteriormente, o aluno escolhe a opção "Gerar Script", ilustrada na Figura 6. Quando o aluno escolhe esta opção, os agentes são acionados, o script na linguagem OTCL é gerado, o cenário é validado e a simulação executada.

Através da simulação dos principais equipamentos de redes o Laboratório Virtual permite a confecção de cenários completos apenas com o principio de arrastar e soltar, destacando-se as seguintes funcionalidades: 


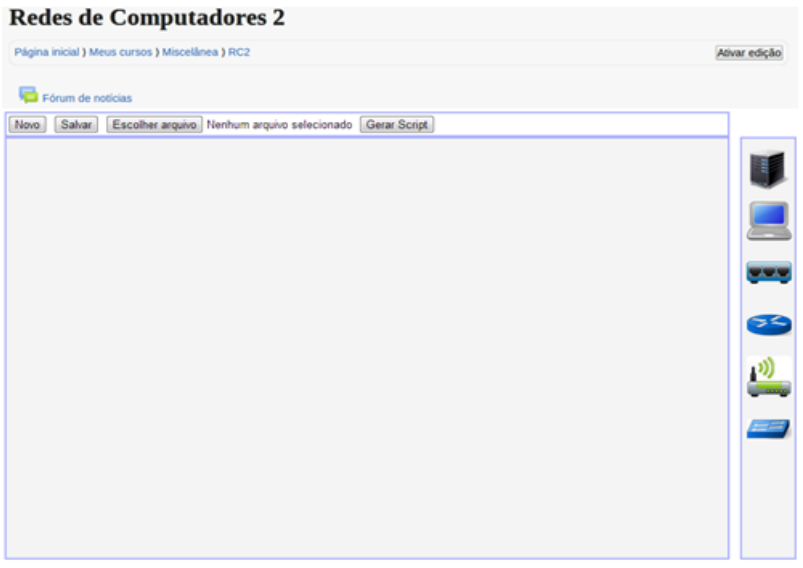

Figura 4. LARC inserido no Moodle

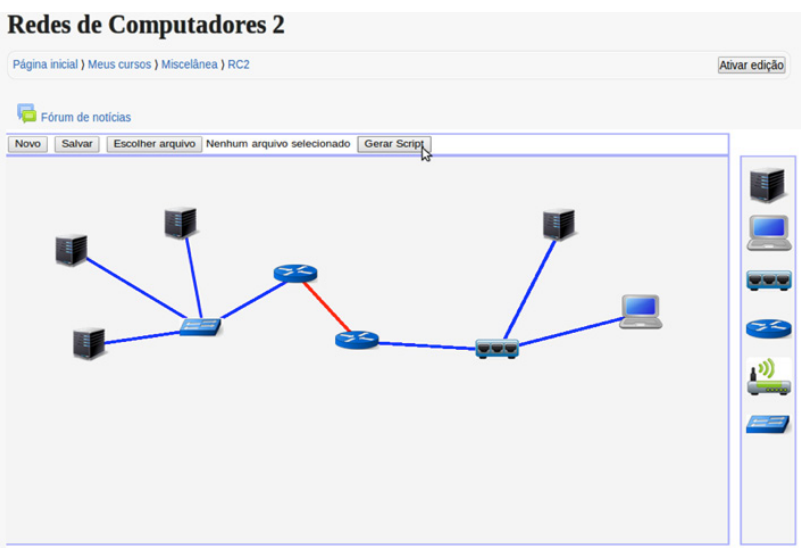

Figura 5. Criando cenário com o LARC

Redes de Computadores 2

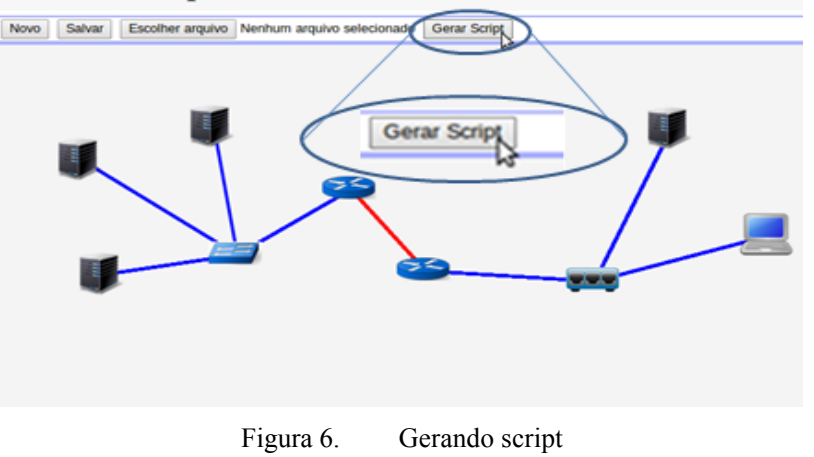

- Criação de topologias com inserção de nós e enlaces;

- Configuração de tráfego com os protocolos TCP e UDP;

- Configuração de aplicações FTP e Telnet;

- Roteamento unicast e multicast;

- Simulação de redes LANs, MANs e WANs;

- Simulação dos cenários confeccionados sem intervenção do NAM; e

- Configuração de protocolos de redes wireless.

Para coleta dos primeiros resultados foi realizada uma conversa informal com 14 professores da área de redes de computadores e usuários do NS2, onde foi apresentada a proposta do LARC e seus principais objetivos. Por meio dessa conversa, foram obtidos os seguintes resultados mostrados na Tabela I.
TABELA I.

RESULTADOS INICIAIS

\begin{tabular}{|c|l|}
\hline Porcetagem & \multicolumn{1}{|c|}{ Significado } \\
\hline $100 \%$ & $\begin{array}{l}\text { Professores e usuários que concordam que o } \\
\text { Laboratório Virtual reduz a sobrecarga do aluno } \\
\text { pela geração de scripts na linguagem OTCL. }\end{array}$ \\
\hline $100 \%$ & $\begin{array}{l}\text { Professores e usuários que concordam que a } \\
\text { visualização no browser da animação é mais } \\
\text { atrativa do que a visualização por meio do } \\
\text { NAM. }\end{array}$ \\
\hline $85,7 \%$ & $\begin{array}{l}\text { Professores e usuários que concordam que o } \\
\text { Laboratório Virtual pode ajudar a fornecer um } \\
\text { feedback mais rápido ao aluno. }\end{array}$ \\
\hline $28,5 \%$ & $\begin{array}{l}\text { Professores e usuários que fizeram observações } \\
\text { sobre a legibilidade do código. }\end{array}$ \\
\hline $92,8 \%$ & $\begin{array}{l}\text { Professores e usuários que utilizariam o Labora- } \\
\text { tório Virtual. }\end{array}$ \\
\hline $92,8 \%$ & $\begin{array}{l}\text { Professores e usuários que concordam que a } \\
\text { utilização do Laboratório Virtual em um AVA } \\
\text { proporciona uma boa ferramenta para cursos da } \\
\text { EaD. }\end{array}$ \\
\hline
\end{tabular}

\section{CONSIDERAÇÕES FINAIS E TRABALHOS FUTUROS}

Através da utilização do Laboratório Virtual apresentado é possível: verificar o funcionamento dos principais protocolos de roteamento; definir diferentes topologias; configurar os equipamentos envolvidos na comunicação entre redes diferentes; diagnosticar e definir soluções para problemas comuns em ambientes reais.

Além disso, o Laboratório Virtual proporciona ao aluno um ambiente atrativo e com baixo custo na implantação pela escolha das ferramentas utilizadas (Moodle e o NS2), facilitando o aprendizado e a utilização do NS2 por meio da geração de scripts na linguagem OTCL.

Como trabalhos futuros espera-se realizar testes no Laboratório Virtual com grupos de alunos e, também, utilizar mais recursos do simulador NS2, possibilitando a criação de cenários de redes de sensores sem fio.

Como o Laboratório Virtual faz uso de sistemas multiagentes, futuramente espera-se implementar nos agentes a capacidade de avaliar o desenvolvimento do aluno na disciplina de redes, identificando suas principais deficiências.

\section{REFERÊNCIAS}

[1] J. Coelho Neto, M. S. Bernadelli, M. P. Pessoa, S. Guilhem, A. Malucelli, and S. Reinehr, "O uso das Tic na formação de professors de escolas que obtiveram baixo IDEB," in: XXII Simpósio Brasileiro de Informática na Educação - SBIE, p.988-996, 2011.

[2] J. C. GLUZ, C. GALAFASSI, F. PENTEADO, "Suporte Técnico/Pedagógico aos OAs pelos AVAs: uma Análise Comparativa das Pesquisas Recentes". In: XXII Simpósio Brasileiro de Informática na Educação - SBIE. Aracajú - SE. p. 294-303. 2011.

[3] J. O. ARIEIRA, C. R. D. ARIEIRA, J. P. A. FUSCO, J. B. SACOMANO, M. O. P. BETTEGA, "Avaliação do aprendizado via educação a distância: a visão dos discentes". Ensaio: aval.pol.públ.Educ. [online]. 2009, vol.17, n.63, pp. 313-340. ISSN 0104-4036.

[4] D. PIVA JÚNIOR, J. R. S. PUPO, L. GAMEZ, "EAD na prática: planejamento, métodos e ambientes de educação online". Rio de Janeiro: Elsevier. 2011.

[5] E. M. H. AMARAL, B. AVILA, H. ZEDNIK, L. TAROUCO, "Laboratório Virtual de Aprendizagem: Uma Proposta Taxonômica"; Revista Novas Tecnologias na Educação - RENOTE, Porto Alegre, v. $9 n^{\circ}$. 2, dezembro, 2011.

[6] M. P. TELES, M. A. S. RIBEIRO JR, M. M. COUTINHO, "GENESI - Gerador de Código para o Network Simulator". Disponí- 
vel em: <www.margalho.pro.br/artigos/genesi.pdf>. Acesso em: ago. 2013.

[7] A. A. A. PONTES, R. W. LIMA, "NsGraph: Interface Gráfica de Modelagem e Geração Automática de Scripts para o NS-2". Anais do XXVI Congresso da SBC. Campo Grande: SBC, 2006.

[8] Site da ferramenta TCLGEN. Disponivel em: $<$ http://www.fccn.pt/crc2003/v3/sessao4/poster09.pdf>. Acesso em: 28 mar. 2013.

[9] F. BELLIFEMINE, G. CAIRE, D. GREENWOOD, "Multi-Agent Systems with JADE". Editora WILEY, 2007; ISBN: 978-0-47005747-6. http://dx.doi.org/10.1002/9780470058411

[10] D. M. OLIVEIRA, R. S. CRUZ, R. J. P. B. SAlguEIRO, T. ROCHA, "An integrated development environment for the NS-2 Network Simulator”. Revista Scientita Plena vol. 8, num. 3, 2012.

\section{AUTORES}

K. H. A. Ferreira faz parte do Grupo de Pesquisa de Redes de Computadores, Sistemas Distribuídos e Multimídia da Universidade do Estado do Rio Grande do Norte - UERN e da Universidade Federal Rural do Semi-Árido - UFERSA, e-mail: (karlhansimuller@gmail.com).
R. W. de Lima faz parte do Grupo de Pesquisa de Redes de Computadores, Sistemas Distribuídos e Multimídia da Universidade do Estado do Rio Grande do Norte UERN, (rommel.lima@gmail.com).

J. O. M. Chaves faz parte do Grupo de Pesquisa de Redes de Computadores, Sistemas Distribuídos e Multimídia da Universidade do Estado do Rio Grande do Norte - UERN e da Universidade Federal Rural do Semi-Árido - UFERSA, (e-mail: oswaldo.mesquita@gmail.com).

M. V. A. de Lima faz parte do Grupo de Pesquisa de Redes de Computadores, Sistemas Distribuídos e Multimídia da Universidade do Estado do Rio Grande do Norte - UERN e da Universidade Federal Rural do Semi-Árido - UFERSA, e-mail: (marcos.engsoft@gmail.com).

Este trabalho teve apoio da Coordenação de Aperfeiçoamento de Pessoal de Nível Superior (CAPES). Submitted 19 March 2014. Published as re-submitted by the authors 05 April 2014. 